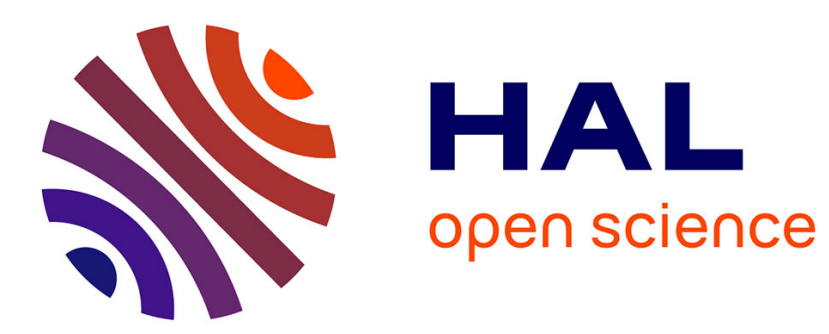

\title{
Imperfect Credibility versus No Credibility of Optimal Monetary Policy
}

Jean-Bernard Chatelain, Kirsten Ralf

\section{To cite this version:}

Jean-Bernard Chatelain, Kirsten Ralf. Imperfect Credibility versus No Credibility of Optimal Monetary Policy. 2018. halshs-01849864

\section{HAL Id: halshs-01849864 \\ https://shs.hal.science/halshs-01849864}

Preprint submitted on 26 Jul 2018

HAL is a multi-disciplinary open access archive for the deposit and dissemination of scientific research documents, whether they are published or not. The documents may come from teaching and research institutions in France or abroad, or from public or private research centers.
L'archive ouverte pluridisciplinaire HAL, est destinée au dépôt et à la diffusion de documents scientifiques de niveau recherche, publiés ou non, émanant des établissements d'enseignement et de recherche français ou étrangers, des laboratoires publics ou privés. 


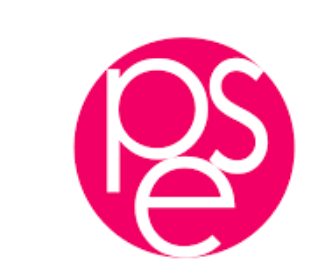

PARISSCHOOL OF ECONOMICS
ECOLE D'ECONOMIE DE PARIS

WORKING PAPER Nº 2018 - 39

\title{
Imperfect Credibility versus No Credibility of Optimal Monetary Policy
}

\author{
Jean-Bernard Chatelain \\ Kirsten Ralf
}

JEL Codes: C61, C62, E31, E52, E58

Keywords : Ramsey optimal policy under imperfact commitment, zerocredibility policy, Impulse response function, Welfare, New-Keynesian Phillips curve






\title{
Imperfect Credibility versus No Credibility of Optimal Monetary Policy.
}

\author{
Jean-Bernard Chatelain* Kirsten Ralf ${ }^{\dagger}$
}

July 25,2018

\begin{abstract}
When the probability of not reneging commitment of optimal monetary policy under quasi-commitment tends to zero, the limit of this equilibrium is qualitatively and quantitatively different from the discretion equilibrium assuming a zero probability of not reneging commitment for the classic example of the new-Keynesian Phillips curve. The impulse response functions and welfare are different. The policy rule parameter have opposite signs. The inflation auto-correlation parameter crosses a saddlenode bifurcation when shitfing to near-zero to zero probability of not reneging commitment. These results are obtained for all values of the elasticity of substitution between goods in monopolistic competition which enters in the welfare loss function and in the slope of the new-Keynesian Phillips curve.
\end{abstract}

JEL classification numbers: C61, C62, E31, E52, E58.

Keywords: Ramsey optimal policy under imperfact commitment, zero-credibility policy, Impulse response function, Welfare, New-Keynesian Phillips curve.

\section{Introduction}

The degree of credibility of policy makers, measured by their probability of not reneging their commitment, is a key determinant of the efficiency of stabilization policy. This paper shows that when the probability of not reneging commitment of optimal monetary policy under quasi-commitment (Schaumburg and Tambalotti (2007), Debortoli and Nunes (2014)) tends to zero, the limit of this equilibrium is different from the discretion equilibrium assuming a zero probability of not reneging commitment ("discretionary policy" according to Gali (2015)) for the classic example of the new-Keynesian Phillips curve. These results are obtained when varying the elasticity of substitution between goods in monopolistic competition which enters into the slope of the new-Keynesian Phillips curve and the welfare loss function.

The impulse response functions and welfare are different. The policy rule parameter have opposite signs. The initial anchor (or jump) of inflation are different. The inflation auto-correlation (or growth factor) parameter crosses a saddle-node bifurcation when shifting from near-zero to zero probability of not reneging commitment. This is a major qualitative change of stability properties of the economy dynamic system with respect

\footnotetext{
*Paris School of Economics, Université Paris 1 Pantheon Sorbonne, PjSE, 48 Boulevard Jourdan, 75014 Paris. Email: jean-bernard.chatelain@univ-paris1.fr

${ }^{\dagger}$ ESCE International Business School, 10 rue Sextius Michel, 75015 Paris, Email: Kirsten.Ralf@esce.fr.
} 
to the robustness to misspecification of stabilization policy. As soon as there is a slight imperfect knowledge of structural parameters by the private sector and by the policy maker, the initial jump of inflation leads to inflation or deflation spirals with discretion equilibrium with a probability equal to one. By contrast, the welfare loss remains moderate for optimal monetary policy under commitment, which leans against inflation spirals even with the same imperfect knowledge of some structural parameters than inflation spirals originated by the complete lack of credibility of the central bank.

Section 2 presents Ramsey optimal policy under imperfect commitment and discretionary policy. Section 3 computes eigenvalues, policy rule parameters, initial anchors of inflation on the cost-push shock, impulse response functions, welfare and robustness to misspecification, in particular for the limit case of near-zero probability versus zero probability of not reneging commmitment. The last section concludes.

\section{Limited Credibility versus Zero Credibility For Ever}

\subsection{New-Keynesian Phillips Curve}

The reference new-Keynesian Phillips curve is the monetary policy transmission mechanism (Gali (2015)):

$$
\pi_{t}=\beta E_{t}\left[\pi_{t+1}\right]+\kappa x_{t}+u_{t} \text { where } \kappa>0,0<\beta<1
$$

where $x_{t}$ represents the welfare-relevant output gap, i.e. the deviation between (log) output and its efficient level. $\pi_{t}$ denotes the rate of inflation between periods $t-1$ and $t$. $u_{t}$ denotes a cost-push shock. $\beta$ denotes the discount factor. $E_{t}$ denotes the expectation operator. The cost push shock $u_{t}$ includes an exogenous auto-regressive component:

$$
u_{t}=\rho u_{t-1}+\eta_{u, t} \text { where } 0<\rho<1 \text { and } \eta_{u, t} \text { i.i.d. normal } N\left(0, \sigma_{u}^{2}\right)
$$

Gali (2015) chooses $\rho=0.8$ for the calibration of the auto-correlation of the costpush shock. The disturbances $\eta_{u, t}$ are identically and independently distributed (i.i.d.) according to a normal distribution with constant variance $\sigma_{u}^{2}$.

The reduced-form parameter (denoted $\kappa$ ) of the slope of the new-Keynesian Phillips curve relates inflation to marginal cost or to the output gap. It is a non-linear function of four preferences and two technology parameters:

$$
\begin{aligned}
\lim _{\varepsilon \rightarrow+\infty} \kappa & =0<\kappa=\left(\sigma+\frac{\varphi+\alpha_{L}}{1-\alpha_{L}}\right) \frac{(1-\theta)(1-\beta \theta)}{\theta} \frac{\left(1-\alpha_{L}\right)}{\left(1-\alpha_{L}+\alpha_{L} \varepsilon\right)}<\kappa_{\max }=\lim _{\varepsilon \rightarrow 1^{+}} \kappa \\
\text { with } \varepsilon & >1,0<\beta, \alpha_{L}, \theta<1, \sigma>0, \varphi>0 . \\
\kappa_{\max } & =\lim _{\varepsilon \rightarrow 1^{+}} \kappa=\left(\sigma+\frac{\varphi+\alpha_{L}}{1-\alpha_{L}}\right) \frac{(1-\theta)(1-\beta \theta)}{\theta}\left(1-\alpha_{L}\right)
\end{aligned}
$$

Gali's (2015, chapter 3) calibration of structural parameter is as follows. The representative household discount factor $\beta=0.99$. It is also assumed $\sigma=1$ (log utility) and $\varphi=1$ (a unitary Frisch elasticity of labor supply). For the production functions of the firms, the measure of decreasing returns to scale of labor is $0<\alpha_{L}=1 / 3<1$ (the production function is $Y=A_{t} L^{1-\alpha_{L}}$ where $Y$ is output, $L$ is labor, $A_{t}$ represents the level of technology and $1-\alpha_{L}=2 / 3$ ). The proportion of firms who do not reset their price each 
period $0<\theta=2 / 3<1$ which corresponds to an average price duration of three quarters. The household's elasticity of substitution between each differentiated intermediate goods is $\varepsilon=6>1$, which is assumed to be larger than one. The maximal value of the slope of the new-Keynesian Phillips curve when varying the elasticity of substitution between intermediate goods, $\kappa_{\max }=0.34$ is obtained when the elasticity of substitution tends to one. In what follows, we provide Gali (2015) numerical values besides general solution in order to keep insights on orders of magnitude.

$$
\begin{aligned}
\kappa & =\left(1+\frac{1+\frac{1}{3}}{1-\frac{1}{3}}\right) \frac{\left(1-\frac{2}{3}\right)\left(1-0.99 \frac{2}{3}\right)}{\frac{2}{3}} \frac{\left(1-\frac{1}{3}\right)}{\left(1-\frac{1}{3}+\frac{1}{3} \varepsilon\right)}=\frac{1.02}{2+\varepsilon} \\
\kappa(\varepsilon=6) & =0.1275<\kappa_{\max }=0.34 .
\end{aligned}
$$

\subsection{Welfare loss function}

In a monetary policy regime indexed by $j$, a policy maker has a period loss function $\frac{1}{2}\left(\pi_{t}^{2}+\alpha_{x, j} x_{t}^{2}\right)$. If the policy maker is maximizing welfare, its preferences $\alpha_{x}$ depend on structural parameters of the transmission mechanism (Gali (2015):

$$
\begin{aligned}
& 0<\alpha_{x}=\frac{\kappa}{\varepsilon}=\left(\sigma+\frac{\varphi+\alpha_{L}}{1-\alpha_{L}}\right) \frac{(1-\theta)(1-\beta \theta)}{\theta} \frac{\left(1-\alpha_{L}\right)}{\left(1-\alpha_{L}+\alpha_{L} \varepsilon\right)} \frac{1}{\varepsilon}<\kappa<\kappa_{\text {max }} \\
& \alpha_{x}=\frac{1.02}{2+\varepsilon} \frac{1}{\varepsilon}=0.02125<\text { if } \varepsilon=6
\end{aligned}
$$

For example, with Gali's (2015) calibration of structural parameters: $\kappa=\frac{1.02}{2+\varepsilon}$ and $\varepsilon=$ 6 , the relative weight of the variance of the policy instrument (output gap) is a very low proportion $(2.125 \%)$ of the weight on the variance of the policy target (inflation). This is a very low relative cost of changing the policy instrument which implies a fast convergence of the policy target. Both the slope $\kappa$ of the monetary transmission mechanism and the policy maker's preferences are decreasing functions of the household's elasticity of substitution between each differentiated goods, whose values varies in $\varepsilon \in] 1,+\infty$ [ (figures 1 and 2).

Figure 1: Slope of the new-Keynesian Phillips curve (solid curve, $\kappa=\frac{1.02}{2+\varepsilon}$ ) and relative welfare cost of changing the policy instrument (dash curve, $\alpha_{x}=\frac{1.02}{2+\varepsilon} \varepsilon$ ) as a function of the elasticity of substitution between differentiated goods.

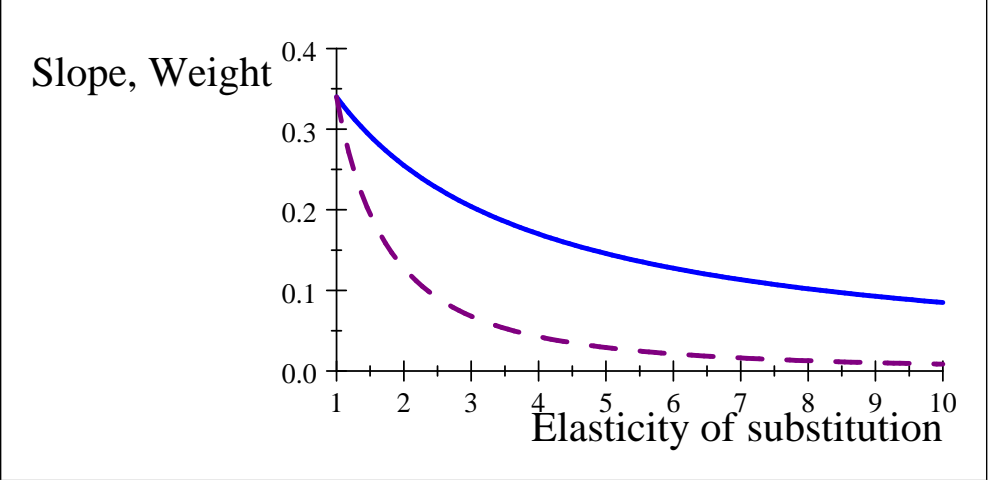

When the elasticity of substitution tends to one, the slope of the new Keynesian Phillips curve is equal to the relative welfare weight on output gap in proportion of the weight on the variance of the policy target (inflation) in the loss function at its maximal 
value $\left(\kappa_{\max }=0.34=\alpha_{x, \max }\right.$ with Gali's calibration $)$.

When the elasticity of substitution of differentiated goods tends to infinity (all other parameters being unchanged), the convergence to zero of the relative welfare weight of the policy instrument in the loss function is faster than the one of the slope of the newKeynesian Phillips curve.

\subsection{Limited Credibility with Ramsey optimal policy under quasi- commitment}

In a monetary policy regime indexed by $j$, a policy maker may re-optimize on each future period with exogenous probability $1-q$ strictly below one ("quasi commitment" by Schaumburg and Tambalotti, 2007 and Debortoli and Nunes, 2014)). Following Schaumburg and Tambalotti (2007), we assume that the mandate to minimize the loss function is delegated to a sequence of policy makers with a commitment of random duration. The degree of credibility is modelled as if it is a change of policy-maker with a given probability of reneging commitment and re-optimizing optimal plans. The length of their tenure or "regime" depends on a sequence of exogenous i.i.d. Bernoulli signals $\left\{\eta_{t}\right\}_{t>0}$ with $E_{t}\left[\eta_{t}\right]_{t \geq 0}=1-q$, with $0<q<1$. If $\eta_{t}=1$, a new policy maker takes office at the beginning of time $t$. Otherwise, the incumbent stays on. A higher probability $q$ can be interpreted as a higher credibility. As seen below, this leads to use a "credibility adjusted" discount factor $\beta q$ in the policy maker's optimal behavior.

Secondly, in this new monetary policy regime indexed by $k$, there may be a switch of the transmission mechanism parameter (the slope of the Phillips curve) $\kappa_{k}$ including in particular, a switch of the representative household's elasticity of substitution between each differentiated goods $\varepsilon_{k}$. This implies a switch of welfare preferences $\alpha_{x, j}=\frac{\kappa_{j}\left(\varepsilon_{j}\right)}{\varepsilon_{j}}$.

Because structural parameters may change for a new regime $k$, long run equilibrium values may also change. Under regime $j$, policy plans solve the following problem (omitting subscript $j$ for the central bank preferences $\alpha_{x}$, transmission mechanism parameter $\kappa$, the auto-correlation of the cost-push shock $\rho$ and its variance of its disturbances $\varepsilon_{t}$ ):

$$
\begin{aligned}
V^{j k}\left(u_{0}\right) & =-E_{0} \sum_{t=0}^{t=+\infty}(\beta q)^{t}\left[\frac{1}{2}\left(\pi_{t}^{2}+\frac{\kappa}{\varepsilon} x_{t}^{2}\right)+\beta(1-q) V^{j k}\left(u_{t}\right)\right] \\
\text { s.t. } \pi_{t} & \left.=\kappa x_{t}+\beta q E_{t} \pi_{t+1}+\beta(1-q) E_{t} \pi_{t+1}^{k}+u_{t} \text { (Lagrange multiplier } \gamma_{t+1}\right) \\
u_{t} & =\rho u_{t-1}+\eta_{u, t}, \forall t \in \mathbb{N}, u_{0} \text { given. }
\end{aligned}
$$

The utility the central bank obtains is next period objectives change is denoted $V^{j k}$. Since when objectives change, the central bank loses its commitment, this value function depends on the policies of the alternative regime. Inflation expectations are an average between two terms. The first term, with weight $q$ is the inflation that would prevail under the current regime upon which there is commitment. The second term with weight $1-q$ is the inflation that would be implemented under the alternative regime, which is taken as given by the current central bank. The key change is that the narrow range of values for the discount factor around 0.99 for quarterly data (4\% discount rate) is much wider for the "credibility weighted discount factor" of the policy maker: $\beta q \in] 0,0.99]$.

Differentiating the Lagrangian with respect to the policy instrument (output gap $x_{t}$ ) and to the policy target (inflation $\pi_{t}$ ) yields the first order conditions: 


$$
\left\{\begin{array} { c } 
{ \frac { \partial \mathcal { L } } { \partial \pi _ { t } } = 0 : \pi _ { t } + \gamma _ { t + 1 } - \gamma _ { t } = 0 } \\
{ \frac { \partial \mathcal { L } } { \partial x _ { t } } = 0 : \frac { \kappa } { \varepsilon } x _ { t } - \kappa \gamma _ { t + 1 } = 0 }
\end{array} \Rightarrow \left\{\begin{array}{c}
x_{t}=x_{t-1}-\varepsilon \pi_{t} \\
x_{t}=\varepsilon \gamma_{t+1}=\varepsilon\left(\gamma_{t}-\pi_{t}\right)
\end{array}\right.\right.
$$

that must hold for $t=1,2, \ldots$ The central bank's Euler equation $\left(\frac{\partial \mathcal{L}}{\partial \pi_{t}}=0\right)$ links recursively the future or current value of central bank's policy instrument $x_{t}$ to its current or past value $x_{t-1}$, because of the central bank's relative cost of changing her policy instrument is strictly positive $\alpha_{x}=\frac{\kappa}{\varepsilon}>0$. This non-stationary Euler equation adds an unstable eigenvalue in the central bank's Hamiltonian system including three laws of motion of one forward-looking variable (inflation $\pi_{t}$ ) and of two predetermined variables $\left(u_{t}, x_{t}\right)$ or $\left(u_{t}, \gamma_{t}\right)$.

The natural boundary condition $\gamma_{0}=0$ minimizes the loss function with respect to inflation at the initial date:

$$
\gamma_{0}=0 \Rightarrow x_{-1}=-\varepsilon \gamma_{0}=0 \text { so that } \pi_{0}=-\frac{1}{\varepsilon} x_{0} \text { or } x_{0}=-\varepsilon \pi_{0}
$$

It predetermines the policy instrument which allows to anchor the forward-looking policy target (inflation). The inflation Euler equation corresponding to period 0 is not an effective constraint for the central bank choosing its optimal plan in period 0 . The former commitment to the value of the policy instrument of the previous period $x_{-1}$ is not an effective constraint. The policy instrument is predetermined at the value zero $x_{-1}=0$ at the period preceding the commitment. Combining the two first order conditions to eliminate the Lagrange multipliers yields the optimal initial anchor of forward inflation $\pi_{0}$ on the predetermined policy instrument $x_{0}$.

Ljungqvist and Sargent (2012, chapter 19) seek the stationary equilibrium process using the augmented discounted linear quadratic regulator solution of the Hamiltonian system (Hansen and Sargent (2007)) as an intermediate step (Chatelain and Ralf (2017) algorithm). This amount to seek a stable subspace of dimension two in a system of three equations including the marginal condition on the policy instrument (or on the Lagrange multiplier on inflation). The policy instrument is exactly correlated with private sectors variables:

$$
x_{t}=F_{\pi} \pi_{t}+F_{u} u_{t} .
$$

with solutions (see appendix) followed by their values using Gali (2015) calibration $(\beta=0.99$ and $q=1)$ :

$$
\begin{aligned}
F_{\pi} & =\left(\frac{\lambda}{1-\lambda}\right) \varepsilon=4.58 \text { and } F_{u}=\frac{1}{\beta q \rho \lambda-1} F_{\pi}=-1.51 F_{\pi}=-6.83 \\
\lambda & =\frac{1}{\beta q}-\frac{\kappa}{\beta q} F_{\pi}=\frac{1}{2}\left(1+\frac{1}{\beta q}+\frac{\varepsilon \kappa}{\beta q}\right)-\sqrt{\frac{1}{4}\left(1+\frac{1}{\beta q}+\frac{\varepsilon \kappa}{\beta q}\right)^{2}-\frac{1}{\beta q}}=0.43
\end{aligned}
$$

We denote the inflation eigenvalue $\lambda$ instead of $\delta$ in Gali (2015). It is the solution of the following characteristic polynomial:

$$
\lambda^{2}-\left(1+\frac{1}{\beta q}+\frac{\kappa \varepsilon}{\beta q}\right) \lambda+\frac{1}{\beta q}=0
$$

The optimal stable dynamics in dimension two are given by (adding Gali (2015) calibration $\beta=0.99$ and $q=1$ ): 


$$
\begin{aligned}
& \left(\begin{array}{c}
E_{t}\left[\pi_{t+1}\right] \\
u_{t}
\end{array}\right)=\left(\begin{array}{cc}
\frac{1}{\beta q}-\frac{\kappa}{\beta q} F_{\pi}=\lambda & -\frac{1}{\beta q}-\frac{\kappa}{\beta q} F_{u} \\
0 & \rho
\end{array}\right)\left(\begin{array}{l}
\pi_{t} \\
u_{t}
\end{array}\right)+\left(\begin{array}{l}
0 \\
1
\end{array}\right) \eta_{t} \\
& \left(\begin{array}{c}
E_{t}\left[\pi_{t+1}\right] \\
u_{t}
\end{array}\right)=\left(\begin{array}{cc}
0.43 & -0.13 \\
0 & 0.8
\end{array}\right)\left(\begin{array}{l}
\pi_{t} \\
u_{t}
\end{array}\right)+\left(\begin{array}{l}
0 \\
1
\end{array}\right) \eta_{t}
\end{aligned}
$$

The dynamics are unique with an initial optimal anchor of forward-looking inflation on the cost-push forcing variable, which is enforced by the optimal initial anchor of inflation on the policy instrument $\pi_{0}=-\frac{1}{\varepsilon} x_{0}$. This optimal anchor rules out sunspot equilibria:

$$
\begin{aligned}
& \pi_{0}=\frac{\lambda}{1-\beta q \rho \lambda} u_{0}=\frac{-F_{u}}{F_{\pi}+\varepsilon} u_{0} \text { because } \\
& x_{0}=F_{\pi} \pi_{0}+F_{u} u_{0} \text { and } \pi_{0}=-\frac{1}{\varepsilon} x_{0}
\end{aligned}
$$

The policy instrument (output gap) (which can be substituted by the Lagrange multiplier of inflation) is optimally predetermined. The auto-regressive cost-push forcing variable is also predetermined. The optimal solution of the Hamiltonian system indeed satisfies Blanchard and Kahn (1980) determinacy condition with two stable eigenvalues: the inflation persistence parameter $\lambda$ and the auto-regressive parameter $\rho$ of the cost-push forcing variable.

This closed loop Ramsey optimal policy is in sharp contrast with the open loop dynamic system before policy intervention where the policy rule parameters are set to zero:

$$
\left(\begin{array}{c}
E_{t}\left[\pi_{t+1}\right] \\
u_{t+1}
\end{array}\right)=\left(\begin{array}{cc}
\frac{1}{\beta} & -\frac{1}{\beta} \\
0 & \rho
\end{array}\right)\left(\begin{array}{l}
\pi_{t} \\
u_{t}
\end{array}\right)+\left(\begin{array}{l}
0 \\
1
\end{array}\right) \eta_{t}=\left(\begin{array}{cc}
1.01 & -1.01 \\
0 & 0.8
\end{array}\right)\left(\begin{array}{l}
\pi_{t} \\
u_{t}
\end{array}\right)+\left(\begin{array}{l}
0 \\
1
\end{array}\right) \eta_{t}
$$

The open loop system has an unstable inflation eigenvalue leading to inflation spirals and a large negative correlation of expected future inflation with current cost-push shock. Both effects are hugely dampened because the elasticity of substitution between differentiated goods is large $(\varepsilon=6)$ which implies a small relative cost of changing the policy instrument (output gap) of $2 \%$ of the cost of the volatility of inflation in the loss function.

\subsection{Zero Credibility For Ever}

With quasi-commitment, the probability of not reneging commitment could be infinitely small (near-zero credibility), but it remains strictly positive: for example, $q=10^{-7}>0$ with $q \in] 0,1]$, hence $\beta q \in] 0,0.99]$. An infinite horizon zero-credibility policy holds when the policy maker re-optimizes with certainty for all future periods: $q=0$. This zero-credibility policy is mentioned as "discretionary policy" by Gali (2015).

The central bank minimizes its loss function subject to the new-Keynesian Phillips curve and such that private sector and the central bank policy instrument reacts only to the contemporary predetermined variable $u_{t}$ at all periods $t$ with a perfect correlation. Following Gali (2015), each period the monetary authority is assumed to choose inflation 
and output gap in order to minimize the period losses

$$
\pi_{t}^{2}+\frac{\kappa}{\varepsilon} x_{t}^{2}
$$

subject to the constraint of the new-Keynesian Phillips curve where the expectation of future inflation is taken as given by the policy maker, because it is a function about future policy instruments (output gaps) and future cost-push shocks which cannot be currently influenced by the policy maker who has zero credibility for ever.

$$
\pi_{t}=\kappa x_{t}+\beta E_{t}\left[\pi_{t+1}\right]+u_{t}
$$

The optimality condition implies a policy rule with perfect negative correlation of the policy instrument (output gap) with the policy target (inflation) with constant parameter given by the opposite of the household's elasticity of substitution between goods (with Gali (2015) calibrated value):

$$
x_{t}=-\varepsilon \pi_{t}=-6 \pi_{t} \text { for } t=0,1,2, \ldots \text { with } \varepsilon>1 .
$$

Using this policy rule to substitute for output gap in the new-Keynesian Phillips curve yields the first equation, besides the auto-regressive equation of the cost-push shock:

$$
\left(\begin{array}{c}
E_{t}\left[\pi_{t+1}\right] \\
u_{t}
\end{array}\right)=\left(\begin{array}{cc}
\frac{1}{\beta}+\frac{\kappa}{\beta} \varepsilon & -\frac{1}{\beta} \\
0 & \rho
\end{array}\right)\left(\begin{array}{l}
\pi_{t} \\
u_{t}
\end{array}\right)+\left(\begin{array}{l}
0 \\
1
\end{array}\right) \eta_{t}=\left(\begin{array}{cc}
1.78 & -1.01 \\
0 & 0.8
\end{array}\right)\left(\begin{array}{l}
\pi_{t} \\
u_{t}
\end{array}\right)+\left(\begin{array}{l}
0 \\
1
\end{array}\right) \eta_{t}
$$

Assuming that both the policy instrument and the policy target are forward-looking and that the cost-push shock is the only predetermined variable, Blanchard and Kahn (1980) determinacy condition forces a unique solution which is given by the unique slope of the eigenvectors of the given stable eigenvalue $0<\rho<1$ of the cost-push shock:

$$
\left(\begin{array}{cc}
\frac{1}{\beta}+\frac{\kappa}{\beta} \varepsilon & -\frac{1}{\beta} \\
0 & \rho
\end{array}\right)\left(\begin{array}{c}
\pi_{t} \\
u_{t}
\end{array}\right)=\rho\left(\begin{array}{c}
\pi_{t} \\
u_{t}
\end{array}\right) \Rightarrow\left(\frac{1}{\beta}+\frac{\kappa}{\beta} \varepsilon-\rho\right) \pi_{t}=\frac{1}{\beta} u_{t}
$$

This slope is the parameter of the exact positive correlation between inflation and the cost-push shock:

$$
\pi_{t}=\left(\frac{1}{1+\kappa \varepsilon-\beta \rho}\right) u_{t}=\left(\frac{1}{1-0.99 \cdot 0.8+0.1275 \cdot 6}\right) u_{t}=1.028 u_{t}
$$

Combining this equation with the policy rule leads to the exact negative correlation between output gap and the cost-push shock is:

$$
x_{t}=-\varepsilon\left(\frac{1}{1+\kappa \varepsilon-\beta \rho}\right) u_{t}=-6.166 u_{t}
$$

The policy maker lets the output gap and inflation deviate from their targets in exact proportion of the current value of the cost-push shock.

The expected loss function is for zero probability of not reneging commitment $(q=0)$ with numerical result for Gali's calibration and impulse response functions $\left(u_{0}=1\right)$ : 


$$
\begin{aligned}
& W(q=0)=-\frac{1}{2} \sum_{t=0}^{t=+\infty} \beta^{t}\left(\pi_{t}^{2}+\frac{\kappa}{\varepsilon} x_{t}^{2}\right)=-\frac{1}{2}\left(1+\frac{\kappa}{\varepsilon} \varepsilon^{2}\right)\left(\frac{1}{1+\kappa \varepsilon-\beta \rho}\right)^{2} \sum_{t=0}^{t=+\infty} \beta^{t}\left(\rho^{t} u_{0}\right)^{2} \\
& W(q=0)=-\frac{1}{2} \frac{1+\kappa \varepsilon}{(1+\kappa \varepsilon-\beta \rho)^{2}} \frac{u_{0}^{2}}{1-\beta \rho^{2}}=-\frac{1}{2} \cdot 5.09
\end{aligned}
$$

\section{Bifurcation}

\subsection{Inflation eigenvalue}

We demonstrate that shifting from limited credibility to zero credibility implies a saddlenode bifurcation of the dynamic system for the new-Keynesian Phillips curve transmission mechanism. The Lagrange multiplier on forward-looking inflation or the policy instrument is optimally predetermined for Ramsey optimal policy. The policy instrument is forward-looking with infinite horizon zero-credibility policy. This implies an additional stable eigenvalue for Ramsey optimal policy with respect to zero-credibility policy, according to Blanchard and Kahn (1980) determinacy condition.

Proposition 1. Saddle-node bifurcation. There is a saddle-node bifurcation on the inflation eigenvalue when shifting from limited credibility $q \in] 0,1]$ (stable eigenvalue $\lambda$ ) to zero credibility for ever $q=0$ (unstable eigenvalue $\lambda_{Z C}$ ).

Proof: For $\varepsilon \in] 1,+\infty$ [, we seek the limits of $\kappa \varepsilon$ which is an increasing function of $\varepsilon$.

$$
\begin{aligned}
\lim _{\varepsilon \rightarrow 1^{+}} \kappa \varepsilon & =\left(\sigma+\frac{\varphi+\alpha_{L}}{1-\alpha_{L}}\right) \frac{(1-\theta)(1-\beta \theta)}{\theta}\left(1-\alpha_{L}\right)=\kappa_{\max }=0.34 . \\
\lim _{\varepsilon \rightarrow+\infty} \kappa \varepsilon & =\left(\sigma+\frac{\varphi+\alpha_{L}}{1-\alpha_{L}}\right) \frac{(1-\theta)(1-\beta \theta)}{\theta} \frac{\left(1-\alpha_{L}\right)}{\alpha_{L}}=\frac{\kappa_{\max }}{\alpha_{L}}=1.02 \text { with } 0<\alpha_{L}<1 \\
& \Rightarrow \kappa_{\max }<\kappa \varepsilon<\frac{\kappa_{\max }}{\alpha_{L}}
\end{aligned}
$$

Zero-credibility inflation eigenvalue is an increasing function of $\kappa \varepsilon$. Its boundary conditions are:

$$
\begin{aligned}
& 1<\frac{1}{\beta}<\frac{1}{\beta}+\frac{1}{\beta} \kappa_{\max }<\lambda_{Z C}=\frac{1}{\beta}+\frac{1}{\beta} \kappa \varepsilon<\frac{1}{\beta}+\frac{1}{\beta} \frac{\kappa_{\max }}{\alpha_{L}} \\
& 1<1.01<1.35<\lambda_{Z C}=\frac{1}{\beta}+\frac{1}{\beta} \kappa \varepsilon<2.04
\end{aligned}
$$

By contrast, for limited credibility $q \in] 0,1], \lambda$ is obtained solving a linear quadratic regulator model so that the inflation eigenvalue is necessarily within the range $[-1,1]$. However, the unit root case which is not necessarily excluded in the general linear quadratic regulator solution (Hansen and Sargent (2007)). More precisely, for the newKeynesian Phillips curve transmission mechanism, limited credibility inflation eigenvalue is a decreasing function of $\kappa \varepsilon$, of $\varepsilon$, of $\beta q$ and of $q$. To prove that their is a saddle-node bifurcation when shifting from limited credibility $q \in] 0,1$ ] (stable eigenvalue $\lambda$ ) to zero credibility for ever $q=0$ (unstable eigenvalue $\lambda_{Z C}$ ), it is sufficient to prove: 


$$
\lim _{q \rightarrow 0^{+} \varepsilon \rightarrow 1^{+}} \lambda=\frac{1}{2}\left(1+\frac{1}{\beta q}+\frac{\kappa \varepsilon}{\beta q}\right)-\sqrt{\frac{1}{4}\left(1+\frac{1}{\beta q}+\frac{\kappa \varepsilon}{\beta q}\right)^{2}-\frac{1}{\beta q}}=\frac{1}{1+\kappa_{\max }}<1
$$

That is:

$$
\lim _{q \rightarrow 0^{+}} \frac{1}{2}\left(1+\frac{1}{\beta q}+\frac{1}{\beta q} \kappa_{\max }\right)-\sqrt{\frac{1}{4}\left(1+\frac{1}{\beta q}+\frac{1}{\beta q} \kappa_{\max }\right)^{2}-\frac{1}{\beta q}}=\frac{1}{1+\kappa_{\max }}=0.746<1
$$

because when $q \rightarrow 0^{+}$:

$$
\lambda \sim \frac{1+\kappa}{2 \beta q}\left(1-\sqrt{1-\frac{4 \beta q}{(1+\kappa)^{2}}}\right) \sim \frac{1+\kappa}{2 \beta q} \frac{1}{2} \frac{4 \beta q}{(1+\kappa)^{2}}=\frac{1}{1+\kappa}
$$

One also checks that there is no flip bifurcation within the regimes of limited credibility $q \in] 0,1]$, seeking the lower bound of the inflation eigenvalue:

$$
\begin{aligned}
\lim _{q \rightarrow 1^{-} \varepsilon \rightarrow+\infty} \lambda & =\frac{1}{2}\left(1+\frac{1}{\beta q}+\frac{\kappa \varepsilon}{\beta q}\right)-\sqrt{\frac{1}{4}\left(1+\frac{1}{\beta q}+\frac{\kappa \varepsilon}{\beta q}\right)^{2}-\frac{1}{\beta q}}=\lambda_{\min }>-1 \\
\lambda_{\min } & =\frac{1}{2}\left(1+\frac{1}{\beta}+\frac{1}{\beta} \frac{\kappa_{\max }}{\alpha_{L}}\right)-\sqrt{\frac{1}{4}\left(1+\frac{1}{\beta}+\frac{1}{\beta} \frac{\kappa_{\max }}{\alpha_{L}}\right)^{2}-\frac{1}{\beta}}=0.379>0>-1
\end{aligned}
$$

Hence we demonstrated the saddle-node bifurcation when shifting from limited credibility $q \in] 0,1$ ] (stable eigenvalue $\lambda$ ) to zero credibility for ever $q=0$ (unstable eigenvalue $\left.\lambda_{Z C}\right)$.

$$
0<\lambda_{\min }<\lambda<\frac{1}{1+\kappa_{\max }}<1<\frac{1+\kappa_{\max }}{\beta}<\lambda_{Z C}<\frac{1}{\beta}+\frac{1}{\beta} \frac{\kappa_{\max }}{\alpha_{L}}
$$

QED.

Figure 2: Inflation eigenvalue $\frac{1}{\beta}-\frac{\kappa}{\beta} F_{\pi}$ as functions of the elasticity of substitution between differentiated goods for $\varepsilon \in[0,6]$ in the case where $q=0$ (zero credibility for ever, dash line with its upper asymptote $\lambda_{\min }$ ) and in the case of limited credibility in four cases: $q=0.001$ and $q=10^{-7}$ (overlap on the top solid line below one), $q=0.5$ (intermediate solid line), and finally $q=1$ (bottom solid line, with a dash line below for its bottom asymptote ). The dash line for 1 corresponds to the saddle-node bifurcation value separating discretion eigenvalue from eigenvalues with limited credibility.

Figure 3: Inflation eigenvalue as a decreasing function of credibility for $q \in] 0,1]$ and of the elasticity of substitution between goods for different values: $\varepsilon=1$ (top decreasing line), 6, 20 and finally 100 and $10^{7}$ which overlap on the bottom decreasing line. 

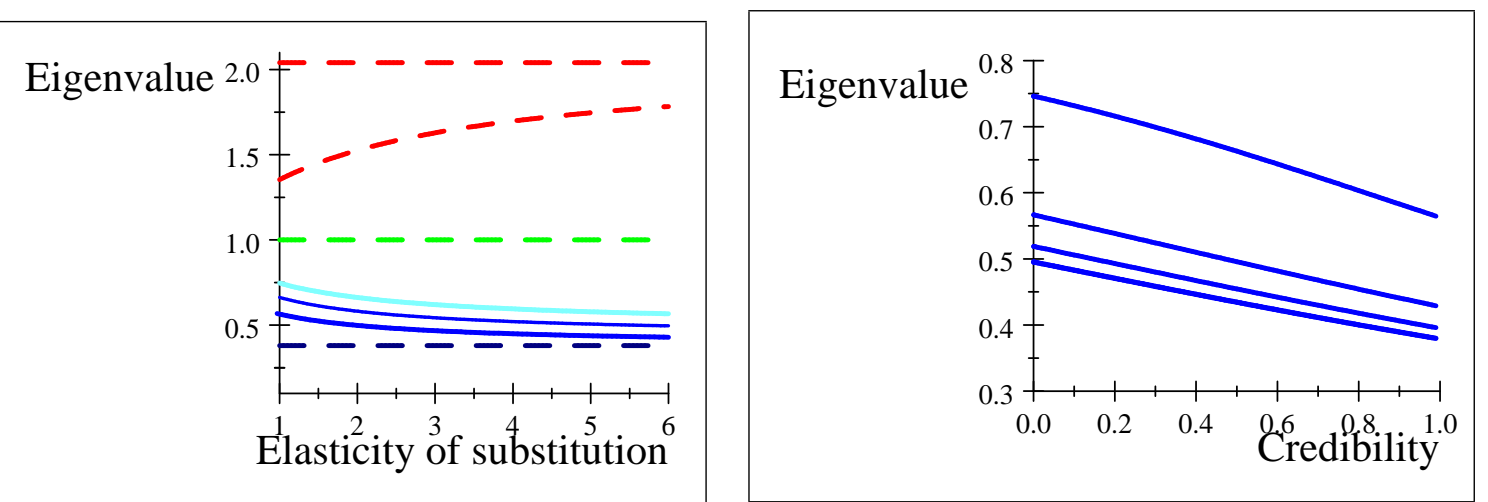

On figures 1 and 2, the limited credibility eigenvalue has an upper bound equal to $\frac{1}{1+\kappa_{\max }}=0.746$ for near zero credibility $q$ and near one elasticity of substitution between goods $\varepsilon$. The larger the credibility $q$, the lower the eigenvalue and the faster the convergence of inflation to equilibrium. The limit eigenvalues obtained with a near-zero probability of not reneging commitment $q=10^{-7}$ are widely different from the eigenvalues obtained with a zero probability of reneging commitment $q=0$ for all values of the elasticity of substitution larger than one.

\subsection{Policy rule response to inflation}

This bifurcation between the case where $q=0$ (zero credibility for ever) versus the case of limited credibility where $q \in] 0,1]$ is caused by opposite feedback mechanism in the policy rule. The inflation rule parameter is an affine and decreasing function of the inflation eigenvalue $\lambda$ according to $\frac{1}{\kappa}-\frac{\beta q}{\kappa} \lambda$ for limited credibility or according to $\frac{1}{\kappa}-\frac{\beta}{\kappa} \lambda_{Z C}$ for zero credibility.

Proposition 2. For limited credibility, the inflation policy rule parameter $F_{\pi}$ is positive. For zero-credibility, the inflation policy rule is negative and below -1 .

Proof. One has:

$$
-\infty<F_{\pi, Z C}=-\varepsilon<-1<0<F_{\pi}
$$

For limited credibility:

$$
-\infty<F_{\pi, Z C}=-\varepsilon<-1<0<F_{\pi}
$$

For limited credibility, the policy rule parameter of the response to inflation is a decreasing function of credibility $q$ and an increasing function of the elasticity of substitution $\varepsilon$. To prove that the policy rule is positive, it is sufficient to prove:

$$
\lim _{q \rightarrow 1^{-}} \lim _{\varepsilon \rightarrow 1^{+}} \frac{1}{\kappa}-\frac{\beta q}{\kappa}\left(\frac{1}{2}\left(1+\frac{1}{\beta q}+\frac{\kappa \varepsilon}{\beta q}\right)-\sqrt{\frac{1}{4}\left(1+\frac{1}{\beta q}+\frac{\kappa \varepsilon}{\beta q}\right)^{2}-\frac{1}{\beta q}}\right)>0
$$

When $q \rightarrow 1^{-}$and when $\varepsilon \rightarrow 1^{+}$

$$
F_{\pi} \sim \frac{1}{\kappa}-\frac{\beta}{\kappa}\left(\frac{1}{2}\left(1+\frac{1}{\beta}+\frac{\kappa}{\beta}\right)-\sqrt{\frac{1}{4}\left(1+\frac{1}{\beta}+\frac{\kappa}{\beta}\right)^{2}-\frac{1}{\beta}}\right)
$$

In this case, one shows in the appendix that $F_{\pi}>0$ is equivalent to $\kappa+\beta>\beta$ which 
is true because $\kappa \rightarrow \kappa_{\max }>0$ (see appendix $4 \mathrm{~A}$ ).

Figure 4. Policy rule parameters for different values of credibility $q$ : 0 (dash line), $10^{-7}$ and $10^{-3}$ (overlap on the upper solid line), 0.5 (intermediate solid line), 1 (bottom solid line).

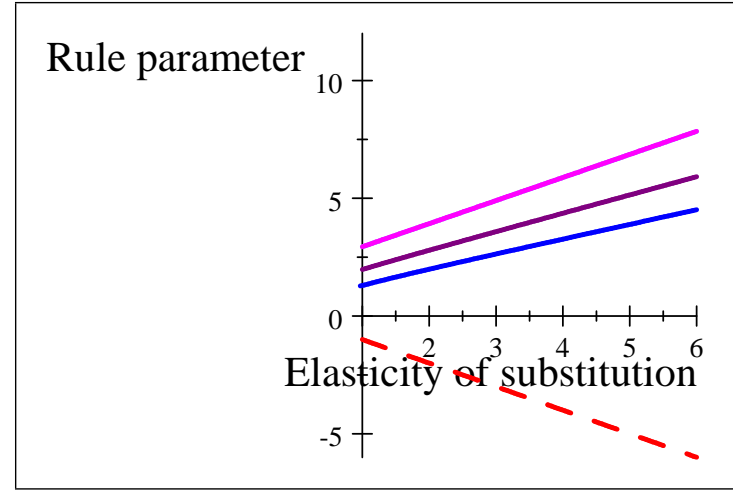

\subsection{Initial anchor of inflation on cost-push shock}

Proposition 3. The initial anchor (or jump) of inflation on the cost-push shock is a decreasing function of the elasticity of substitution between goods for both limited credibility and zero credibility policy regimes. It is an increasing function of the limited credibility of the policy maker.

Proof. Output gap and inflation are exactly linearly related at the initial date $x_{0}=$ $-\varepsilon \pi_{0}$ for limited and zero-credibility case. The anchor of inflation on the cost-push shock are generally different between limited credibility versus zero credibility:

$$
\pi_{0}=\frac{\lambda}{1-\beta q \rho \lambda} u_{0} \text { versus } \pi_{0, Z C}=\frac{1}{1-\beta \rho+\kappa \varepsilon} u_{0}
$$

For zero credibility, the anchor of inflation is a decreasing function of $\kappa \varepsilon$ which is an increasing function of $\varepsilon$. As $\kappa_{\max }<\kappa \varepsilon<\frac{\kappa_{\max }}{\alpha_{L}}$, the zero credibility initial anchor of inflation $\left(\pi_{0} / u_{0}\right)$ is bounded:

$$
0.81=\frac{1}{1-\beta \rho+\frac{\kappa_{\max }}{\alpha_{L}}}<\frac{1}{1-\beta \rho+\kappa \varepsilon}<\lim _{\varepsilon \rightarrow 1} \frac{1}{1-\beta \rho+\kappa \varepsilon}=\frac{1}{1-\beta \rho+\kappa_{\max }}=1.82
$$

For limited credibility, the anchor of inflation is a decreasing function of $\kappa \varepsilon$ which is an increasing function of $\varepsilon$. As $\kappa_{\max }<\kappa \varepsilon<\frac{\kappa_{\max }}{\alpha_{L}}$, the zero credibility initial anchor of inflation $\left(\pi_{0} / u_{0}\right)$ upper bound.

$$
\lim _{q \rightarrow 1^{-\varepsilon \rightarrow 1}} \frac{\lambda}{1-\beta q \rho \lambda}=\lim _{q \rightarrow 1^{-\varepsilon \rightarrow 1}} \frac{\lambda}{1-\beta \rho \lambda}=1.02
$$

With:

$$
\lim _{q \rightarrow 1^{-} \varepsilon \rightarrow 1} \lim _{1} \lambda=\frac{1}{2}\left(1+\frac{1}{\beta}+\frac{\kappa_{\max }}{\beta}\right)-\sqrt{\frac{1}{4}\left(1+\frac{1}{\beta}+\frac{\kappa_{\max }}{\beta}\right)^{2}-\frac{1}{\beta}}=0.56
$$

The initial anchor of near-zero credibility is always strictly smaller than the initial anchor in the case of zero credibility. The gap tends to zero when the auto-correlation of the forcing variable tends to zero and when the elasticity of substitution tends to one: 
$\rho \rightarrow 0$ and $\varepsilon \rightarrow 1$

$$
\lim _{q \rightarrow 0^{+}} \frac{\lambda}{1-\beta q \rho \lambda}=\lim _{q \rightarrow 0^{+}} \lambda \sim \frac{1}{1+\kappa}<\frac{1}{1-\beta \rho+\kappa \varepsilon}
$$

QED.

For Gali (2015) calibration with $\rho=0.8$ (which is far from $\rho \rightarrow 0$ ), for any elasticity of substitution and for any probability of not reneging commitment, the zero credibility initial anchor of inflation is much higher $(+80 \%)$ than the limited credibility initial anchor of inflation (figure 5).

Figure 5: Initial anchor of inflation as a decreasing function of the elasticity of substitution for $q=0$ (dash line top), $q=1$ (solid line, second line from top), $q=0.5$ (solid line, third curve from top), $q=10^{-7}$ with a value equal to the inflation eigenvalue $\lambda$ (solid line, bottom curve) .

Figure 6: Initial anchor of inflation as an increasing function of credibility $q \in] 0,1]$ for $\varepsilon=1$ (top line), 6 (intermediate line) and $10^{7}$ (bottom line).
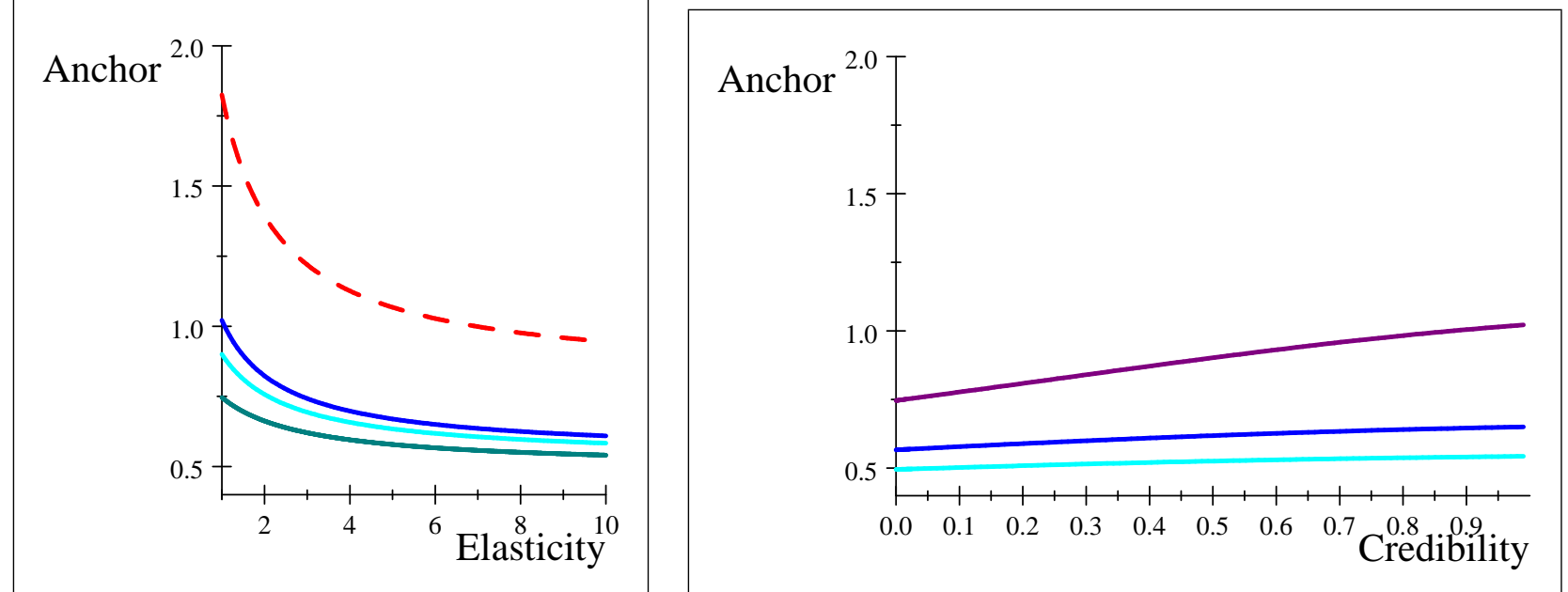

As seen in figure 6, there is a potential trade-off within the cases of limited credibility: more credibility (a higher $q \in] 0,1]$ ) implies faster convergence on subsequent period over a longer expected duration measured by the inflation eigenvalue, but it allows a higher initial anchor of inflation which slows convergence. Which effect offset the other is found computing impulse response functions and welfare losses in the next section.

\subsection{Impulse response functions and welfare}

Impulse response function are shown on figure 7 for four different degrees of credibility $q$ : 0 (for ever discretion), $10^{-7}$ (limit-discretion), 0.5, 1 (infinite horizon commitment). The first impulse response of zero credibility a markedly over the impulse response functions of inflation with limited credibility. This is reflected in the evaluation of the relative welfare loss. Using table 3 formulas, we replicate Gali (2015) impulse response functions for $\varepsilon=6$.

Table 3: Expected impulse response functions.

\begin{tabular}{|l|l|l|l|}
\hline Credibility & Impulse response functions following $u_{0}$ & $F_{\pi}$ & $F_{u}$ \\
\hline$q \in] 0,1]$ & $\left(\begin{array}{l}\pi_{t} \\
u_{t}\end{array}\right)=\left(\begin{array}{cc}\frac{1}{\beta q}-\frac{\kappa}{\beta q} F_{\pi}=\lambda & -\frac{1}{\beta q}-\frac{\kappa}{\beta q} F_{u} \\
0 & \rho\end{array}\right)^{t}\left(\begin{array}{c}\frac{\lambda}{1-\beta q \rho \lambda} \\
1\end{array}\right) u_{0}$ & $\varepsilon \frac{\lambda}{1-\lambda}$ & $\frac{-\lambda}{1-\beta q \rho \lambda} F_{\pi}$ \\
\hline$q=0$ & $\left(\begin{array}{c}\pi_{t} \\
u_{t}\end{array}\right)=\left(\begin{array}{cc}\frac{1}{\beta}+\frac{\kappa}{\beta} \varepsilon & -\frac{1}{\beta} \\
0 & \rho\end{array}\right)^{t}\left(\begin{array}{c}\frac{1}{1-\beta \rho+\kappa \varepsilon} \\
1\end{array}\right) u_{0}$ & $-\varepsilon$ & 0 \\
\hline
\end{tabular}


Welfare losses for different elasticity and different credibility computation are reported in table 4 for Gali (2015) calibration. By contrast to discretion equilibrium, we did not find a closed form formula for welfare losses in the case of limited credibility. Hence, we simulate the model over 200 periods in order to compute welfare. For comparison with the welfare of infinite horizon regimes, the limited credibility welfare is arbitrarily computed using a discount factor of $\beta=0.99$ instead of $\beta q$ in order to take into account in a approximation the regimes which appears with probability $1-q$.

Table 1: Welfare loss in percentage of welfare loss with infinite horizon commitment $\left(w(\beta q)=\frac{W(\beta q)}{W(0.99)}-1, \beta=0.99, q=1\right)$.

\begin{tabular}{|l|l|l|l|l|l|l|l|l|}
\hline$\alpha_{x}$ & $\varepsilon$ & $\kappa=\varepsilon \alpha_{x}$ & $2 W(0.99)$ & $w(0)$ & $w\left(10^{-7}\right)$ & $w(0.1)$ & $w(0.5)$ & $w(0.8)$ \\
\hline $10^{-7}$ & 3193 & 0.00032 & -2.119 & $73 \%$ & $2.1 \%$ & $10.8 \%$ & $6.8 \%$ & $2.8 \%$ \\
\hline 0.02125 & 6 & 0.1275 & -2.688 & $89 \%$ & $0.03 \%$ & $10.9 \%$ & $7.4 \%$ & $3.2 \%$ \\
\hline 0.1 & 2.35 & 0.235 & -3.489 & $111 \%$ & $8.6 \%$ & $10.2 \%$ & $7.8 \%$ & $3.6 \%$ \\
\hline 0.34 & 1 & 0.34 & -7.971 & $141 \%$ & $23.6 \%$ & $7 \%$ & $7.8 \%$ & $4.1 \%$ \\
\hline
\end{tabular}

As seen with the impulse response functions in the case $\varepsilon=6$, the gap between discretion and limited commitment is very large. The loss of welfare by comparison between infinite horizon credibility versus discretion is gigantic ranging from $60 \%$ to $118 \%$ increases of welfare losses.

When considering limited credibility taking into account the probability of not reneging commitment, the losses are relatively modest (at most an increase of $24 \%$ of welfare losses in the limit case of the elasticity of substitution tending to 1) for a wide range of probabilities from $10^{-7}$ to 0.8 . The welfare gap between limited credibility with a probability of not reneging commitment near zero: $q=10^{-7}$ versus $q=0$ for discretion model is gigantic. For welfare evaluation, limited credibility is a very distinct model than zero credibility (discretion).

\subsection{Robustness to misspecification}

We assume that there is a misspecification by the private sector and the policy maker on their exact knowledge of parameters $\beta, \rho, \kappa, \varepsilon, u_{0}$ so that the initial anchor of inflation $\pi_{0}$ deviates from $\pm 10 \%$ with respect to its value with exact knowledge of parameters. This assumption is grounded by a number of major measurement issues:

1. Inflation $\pi_{0}$ is not measured with exact precision. This error is related to consumer price index versus core inflation, quality adjusted bias and the revisions of national accounts.

2. A major source of new-Keynesian uncertainty is the measurement of the unobservable cost-push shock initial value $u_{0}$ depending on its past value $u_{-1}$, on its autocorrelation $\rho$ and on the disturbance $\eta_{0}$. The cost-push shock is indirectly measured an auto-correlated residual. It faces identification issues when an additional lag is included for inflation in hybrid Phillips curve. As a residual, it varies widely depending on misspecfication of inflation dynamics.

3. The estimated slope $\kappa\left(\beta, \varepsilon, \alpha_{L}, \theta, \sigma, \varphi\right)$ of the new-Keynesian Phillips curve in only known with a standard error. It sign is even uncertain (Mavroeidis et al. (2015)). It is itself a function of six not so precisely known structural parameters $\kappa(\beta, \varepsilon, \eta, \theta, \sigma, \varphi)$, in particular the proportion of firms $\theta$ who do not reset their price at each quarter.

4. The elasticity of substitution between differentiated inputs $\varepsilon$ in monopolistic competition which enters into welfare relative weight is not precisely known. Some authors 
may refer to the measurement of Lerner index which are themselves lacking precision, with a calibration of $\varepsilon=11$ instead of Gali (2015) calibration of $\varepsilon=6$.

5 . The policy maker discount factor $\beta$ may vary much more with a adjusted discount factor $\beta q$ depending on the probability $q$ of not reoptimizing. For example, Debortoli and Lakdawala (2016) point estimate is $\widehat{q}=0.81$ in a $95 \%$ confidence interval $[0.777,0.851]$.

Using table 3 formulas, we compute two impulse response functions of out of equilibrium path when facing $\pm 10 \%$ error on the initial anchor of inflation using using Gali's (2015) calibration.

For near zero credibility $\left(q=10^{-7}\right)$, the error gap of $10 \%$ with respect to the perfect knowledge optimal path at the initial date is reduced to less than $1 \%$ after eight quarters (figure 8).

For zero credibility $(q=0)$, the error gap of $10 \%$ with respect to the perfect knowledge optimal path at the initial date is increased to $110 \%$ after four quarters and to $270 \%$ after eight quarters (figure 9) with inflation or deflation spirals. After six quarters, the divergence of inflation reaches $+1 \%$ additional inflation with $+10 \%$ error or $-2 \%$ additional deflation with $-10 \%$ error with respect to the perfect knowledge impulse response function.

In the perfect knowledge case, which has a probability zero for practitioners of stabilization policy, the expected impulse response function may suggest that discretionary policy leans against inflation spirals, while using inflation rule parameters destabilizing the inflation eigenvalue. By contrast, in the imperfect knowledge case with zero credibility, the outcome of discretionary policy is a probability equal to one of inflation or deflation spirals. The core behavioral hypothesis that a policy maker sticks to an exactly zero probability of not reneging commitment for ever is also an assumption with a probability zero for practitioners of stabilization policy.

\subsection{Conclusion}

Even in the most favorable case of an elasticity of substitution between goods tending to one, the limited-credibility equilibrium when the probability to renege commitment tends to zero is never the limit of the zero credibility for ever equilibrium: positive sign versus negative sign of the response of the policy instrument to inflation, stability versus instability of the inflation eigenvalue, small versus large initial anchor of inflation, small versus large magnitude of welfare loss, robustness versus lack of robustness to a large range of misspecification and measurement error.

The bifurcation between zero credibility versus limited credibility is a general result for any linear model of the private sector with any number of lags and any number of variables including at least one forward-looking variable with a policy maker quadratic loss function. With limited credibility, the policy maker's Lagrange multipliers of each private sector forward-looking variables are predetermined variables which are eliminated by assumption in the zero credibility model (e.g. Chatelain and Ralf (2017a)). Hence, Blanchard and Kahn (1980) determinacy condition implies more stable eigenvalues with limited credibility model with respect to zero credibility. For example, including the private sector consumption Euler equation besides the new-Keynesian Phillips curve leads to a Hopf bifurcation (Chatelain and Ralf (2017b)). This also implies that the number of non-collinear variables in a vector auto-regressive representation is smaller with zero credibility than with limited credibility (Chatelain and Ralf (2017c)). Adding more variables, more lags and and more parameters in the transmission mechanism may give the 
illusion that the zero credibility model may fit the data. But this result is obtained in neglecting exact and weak identification issues and the parsimony criterion for the number of parameters when comparing models (Chatelain and Ralf (2018)).

\section{References}

[1] Blanchard O.J. and Kahn C. (1980). The solution of linear difference models under rational expectations. Econometrica, 48, pp. 1305-1311.

[2] Chatelain, J. B., and Ralf, K. (2017a). A Simple Algorithm for Solving Ramsey Optimal Policy with Exogenous Forcing Variables. Paris School of Economics and SSRN working paper.

[3] Chatelain, J. B., and Ralf, K. (2017b). Hopf bifurcation from new-Keynesian Taylor rule to Ramsey optimal policy (2017). Paris School of Economics and SSRN working paper. https://papers.ssrn.com/sol3/papers.cfm?abstract_id=2971227.

[4] Chatelain, J. B., and Ralf, K. (2017c). Can we Identify Fed's preferences? Paris School of Economics and SSRN working paper.

[5] Chatelain, J. B., and Ralf, K. (2018). Publish and Perish: Creative Destruction and Macroeconomic Theory. History of Economic Ideas, 2, forthcoming. Preprint Paris School of Economics and SSRN working paper, version 2.

[6] Debortoli, D., and Nunes, R. (2014). Monetary regime switches and central bank preferences. Journal of Money, Credit and Banking, 46(8), 1591-1626.

[7] Debortoli, D., \& Lakdawala, A. (2016). How credible is the federal reserve? a structural estimation of policy re-optimizations. American Economic Journal: Macroeconomics, 8(3), 42-76.

[8] Fujiwara, I., Kam, T., \& Sunakawa, T. (2016). A note on imperfect credibility. SSRN working paper.

[9] Gali J. (2015). Monetary Policy, Inflation, and the Business Cycle, (2nd edition) Princeton University Press.

[10] Hansen L.P. and Sargent T. (2008). Robustness, Princeton University Press, Princeton.

[11] Ljungqvist L. and Sargent T.J. (2012). Recursive Macroeconomic Theory. 3rd edition. The MIT Press. Cambridge, Massaschussets.

[12] Schaumburg, E., and Tambalotti, A. (2007). An investigation of the gains from commitment in monetary policy. Journal of Monetary Economics, 54(2), 302-324. 


\subsection{Appendix 1: Augmented Discounted Linear Quadratic Reg- ulator}

The new-Keynesian Phillips curve can be written as a function of the Lagrange multiplier where $\kappa>0,0<\beta<1$ and $0<q<1$ (Debortoli and Nunes (2014, appendix A). We keep Gali (2015) chapter $5 \gamma_{t+1}$ notation of the Lagrange multiplier with one step ahead subscript: it corresponds to Debortoli and Nunes (2014) notation $\lambda_{t}$. Our notation for the stable eigenvalue $\lambda$ corresponds to Debortoli and Nunes (2014) notations " $\psi_{y}=1 / \gamma$ ".

$$
E_{t} \pi_{t+1}+\frac{\kappa \varepsilon}{\beta q} \gamma_{t+1}=\frac{1}{\beta q} \pi_{t}-\frac{1}{\beta q} u_{t}-\frac{1-q}{q} E_{t} \pi_{t+1}^{j}
$$

In what follows, $\beta$ refers to $\beta q$ to simplify notations. The solution of the Hamiltonian system are based on the demonstrations of the augmented discounted linear quadratic regulator in Anderson, Hansen, McGrattan and Sargent [1996], following the steps in Chatelain and Ralf (2017a):

$$
\left(\begin{array}{ccc}
1 & \frac{\kappa \varepsilon}{\beta} & 0 \\
0 & 1 & 0 \\
0 & 0 & 1
\end{array}\right)\left(\begin{array}{c}
\pi_{t+1} \\
\gamma_{t+1} \\
u_{t+1}
\end{array}\right)=\left(\begin{array}{ccc}
\frac{1}{\beta} & 0 & \frac{-1}{\beta} \\
-1 & 1 & 0 \\
0 & 0 & \rho
\end{array}\right)\left(\begin{array}{c}
\pi_{t} \\
\gamma_{t} \\
u_{t}
\end{array}\right)+\left(\begin{array}{c}
-\frac{1-q}{q} E_{t} \pi_{t+1}^{j} \\
0 \\
0
\end{array}\right)
$$

The Hamiltonian system is:

$$
\left(\begin{array}{l}
\pi_{t+1} \\
\gamma_{t+1} \\
u_{t+1}
\end{array}\right)=\left(\begin{array}{ccc}
\frac{1}{\beta}+\frac{\kappa \varepsilon}{\beta} & -\frac{\kappa \varepsilon}{\beta} & -\frac{1}{\beta} \\
-1 & 1 & 0 \\
0 & 0 & \rho
\end{array}\right)\left(\begin{array}{c}
\pi_{t} \\
\gamma_{t} \\
u_{t}
\end{array}\right)+\left(\begin{array}{c}
-\frac{1-q}{q} E_{t} \pi_{t+1}^{j} \\
0 \\
0
\end{array}\right)
$$

The characteristic polynomial of this upper square matrix is:

$$
\lambda^{2}-\left(1+\frac{1}{\beta}+\frac{\kappa \varepsilon}{\beta}\right) \lambda+\frac{1}{\beta}=0
$$

The Hamiltonian matrix has two stable roots $\rho$ and $\lambda(\lambda$ is denoted $\delta$ in Gali (2015)) and one unstable root $\frac{1}{\beta \lambda}$. The determinant of the matrix is $\rho \lambda \frac{1}{\beta \lambda}=\rho \frac{1}{\beta}$. Then $\lambda<\sqrt{\frac{1}{\beta}}<$ $\frac{1}{\beta \lambda}$. The trace of the matrix is

$$
\lambda=\frac{1}{2}\left(1+\frac{1}{\beta}+\frac{\kappa \varepsilon}{\beta}-\sqrt{\left(1+\frac{1}{\beta}+\frac{\kappa \varepsilon}{\beta}\right)^{2}-\frac{4}{\beta}}\right)
$$

Policy rule parameter function of $\lambda(\varepsilon)$ and $\varepsilon$ :

$$
\begin{aligned}
(1-\lambda)\left(1-\frac{1}{\beta \lambda}\right) & =-\frac{\kappa \varepsilon}{\beta} \Longrightarrow\left(\frac{1-\lambda}{\beta \lambda}\right)\left(\frac{\beta \lambda-1}{\kappa}\right)=-\frac{\varepsilon}{\beta} \Longrightarrow \\
F_{\pi} & =\frac{1-\beta \lambda}{\kappa}=\left(\frac{\lambda}{1-\lambda}\right) \varepsilon
\end{aligned}
$$

Hamiltonian system function of the stable eigenvalue $\lambda$ (eliminating $\varepsilon$ ): 


$$
\left(\begin{array}{l}
\pi_{t+1} \\
\gamma_{t+1} \\
u_{t+1}
\end{array}\right)=\left(\begin{array}{ccc}
\lambda+\frac{1}{\beta \lambda}-1 & 1+\frac{1}{\beta}-\lambda-\frac{1}{\beta \lambda} & -\frac{1}{\beta} \\
-1 & 1 & 0 \\
0 & 0 & \rho
\end{array}\right)\left(\begin{array}{c}
\pi_{t+1} \\
\gamma_{t+1} \\
u_{t+1}
\end{array}\right)
$$

Proposition A1: Solution of Ricatti and Sylvester equation: Rule parameters $P_{u}$ and $P_{z}$ of the response of the Lagrange multiplier on inflation to exogenous variables:

$$
\begin{aligned}
\gamma_{t} & =P_{\pi} \pi_{t}+P_{u} u_{t} \\
P_{\pi} & =\frac{1}{1-\lambda}>0, P_{u}=\frac{1}{1-\lambda} \frac{\frac{1}{\beta}}{\rho-\frac{1}{\beta \lambda}}=\frac{1}{1-\lambda} \frac{\lambda}{\beta \lambda \rho-1}<0
\end{aligned}
$$

Demonstration: We use the method of undetermined coefficients of Anderson, Hansen, McGrattan and Sargent's (1996), section 5. The solution is the one that stabilizes the state-costate vector for any initialization of inflation $\pi_{0}$ and of the exogenous variables $u_{0}$ in a stable subspace of dimension two within a space of dimension three $\left(\pi_{t}, \gamma_{t}, u_{t}\right)$ of the Hamiltonian system. We seek a characterization of the Lagrange multiplier $\gamma_{t}$ of the form:

$$
\gamma_{t}=P_{\pi} \pi_{t}+P_{u} u_{t}
$$

To deduce the control law associated with vector $\left(P_{\pi}, P_{u}\right)$, we substitute it into the Hamiltonian system:

$$
\begin{aligned}
& \left(\begin{array}{c}
\pi_{t+1} \\
P_{\pi} \pi_{t+1}+P_{u} u_{t+1} \\
u_{t+1}
\end{array}\right) \\
& =\left(\begin{array}{ccc}
\frac{1}{\beta}-(1-\lambda)\left(1-\frac{1}{\beta \lambda}\right) & (1-\lambda)\left(1-\frac{1}{\beta \lambda}\right) & -\frac{1}{\beta} \\
-1 & 1 & 0 \\
0 & 0 & \rho
\end{array}\right)\left(\begin{array}{c}
\pi_{t} \\
P_{\pi} \pi_{t}+P_{u} u_{t} \\
u_{t}
\end{array}\right)
\end{aligned}
$$

We write the last two equations in this system separately:

$$
\begin{aligned}
P_{\pi} \pi_{t+1}+P_{u} u_{t+1} & =\left(P_{\pi}-1\right) \pi_{t}+P_{u} u_{t} \\
u_{t+1} & =\rho u_{t}
\end{aligned}
$$

It follows that:

$$
\pi_{t+1}=\frac{P_{\pi}-1}{P_{\pi}} \pi_{t}+\frac{(1-\rho) P_{u}}{P_{\pi}} u_{t}
$$

The first equation is such that:

$$
\pi_{t+1}=\left[\frac{1}{\beta}-(1-\lambda)\left(1-\frac{1}{\beta \lambda}\right)\right] \pi_{t}+(1-\lambda)\left(1-\frac{1}{\beta \lambda}\right)\left(P_{\pi} \pi_{t}+P_{u} u_{t}\right)-\frac{1}{\beta} u_{t}
$$

Factorizing: 


$$
\pi_{t+1}=\left[\frac{1}{\beta}-(1-\lambda)\left(1-\frac{1}{\beta \lambda}\right)+(1-\lambda)\left(1-\frac{1}{\beta \lambda}\right) P_{\pi}\right] \pi_{t}+\left[(1-\lambda)\left(1-\frac{1}{\beta \lambda}\right) P_{u}-\frac{1}{\beta}\right] u_{t}
$$

The method of undetermined coefficients implies for the first term:

$$
\begin{aligned}
\frac{P_{\pi}-1}{P_{\pi}} & =\frac{1}{\beta}+(1-\lambda)\left(1-\frac{1}{\beta \lambda}\right)\left(P_{\pi}-1\right) \\
P_{\pi} & =\frac{1}{1-\lambda}
\end{aligned}
$$

For the second term:

$$
\begin{aligned}
\frac{(1-\rho) P_{u}}{P_{\pi}} & =(1-\lambda)\left(1-\frac{1}{\beta \lambda}\right) P_{u}-\frac{1}{\beta} \Rightarrow \\
\frac{1}{\beta} & =\left(1-\frac{1}{\beta \lambda}-1+\rho\right)(1-\lambda) P_{u} \Rightarrow \\
P_{u} & =\frac{1}{1-\lambda} \frac{\frac{1}{\beta}}{\rho-\frac{1}{\beta \lambda}} \Rightarrow \frac{P_{u}}{P_{\pi}}=\frac{\frac{1}{\beta}}{\rho-\frac{1}{\beta \lambda}}=\frac{-\lambda}{1-\lambda \beta \rho}
\end{aligned}
$$

QED

Proposition A2: Optimal policy rule parameters formulas:

$$
\begin{aligned}
& F_{\pi}=\varepsilon\left(P_{\pi}-1\right)=\lambda \varepsilon P_{\pi}=\varepsilon \frac{\lambda}{1-\lambda}=\frac{1-\beta \lambda}{\kappa} \\
& F_{u}=\varepsilon P_{u}=\varepsilon P_{\pi} \frac{\lambda}{\beta \lambda \rho-1}=\varepsilon \frac{1}{1-\lambda} \frac{\lambda}{\beta \lambda \rho-1} \\
& \frac{F_{u}}{F_{\pi}}=A=\frac{1}{\lambda} \frac{P_{u}}{P_{\pi}}=\frac{1}{\beta \lambda \rho-1}=\frac{P_{u}}{P_{\pi}-1}=-1+\beta \rho \frac{P_{u}}{P_{\pi}}
\end{aligned}
$$

\section{Demonstration:}

The first order condition relates Lagrange multiplier to the policy instrument:

$$
\begin{aligned}
x_{t} & =\varepsilon \gamma_{t+1}=\varepsilon\left(\gamma_{t}-\pi_{t}\right) \\
x_{t} & =F_{\pi} \pi_{t}+F_{u} u_{t}=\varepsilon\left(\gamma_{t}-\pi_{t}\right)=\varepsilon\left(P_{\pi} \pi_{t}+P_{u} u_{t}-\pi_{t}\right) \Rightarrow \\
F_{\pi} & =\varepsilon\left(P_{\pi}-1\right), F_{u}=\varepsilon P_{u}
\end{aligned}
$$

Proposition A3: From LQR to Gali (2015) vector basis (replace policy target by policy instrument).

One has:

$$
\frac{-1-\kappa F_{u}}{\beta}=\frac{-1-\kappa A \frac{1-\beta \lambda}{\kappa}}{\beta}=\frac{1}{\beta \lambda \rho-1} \lambda-\frac{1}{\beta}-\frac{\frac{1}{\beta \lambda \rho-1}}{\beta}=\frac{(1-\rho) \lambda}{\beta \lambda \rho-1}=(1-\rho) \lambda A
$$


One has:

$$
\begin{gathered}
\left\{\begin{array}{c}
\left(\begin{array}{c}
u_{t+1} \\
\pi_{t+1}
\end{array}\right)=\left(\begin{array}{cc}
\rho & 0 \\
(1-\rho) A \lambda & \lambda
\end{array}\right)\left(\begin{array}{c}
u_{t} \\
\pi_{t}
\end{array}\right)+\left(\begin{array}{c}
\varepsilon_{t} \\
0
\end{array}\right) \\
\left(\begin{array}{c}
u_{t} \\
x_{t}
\end{array}\right)=\left(\begin{array}{cc}
1 & 0 \\
A F_{\pi} & F_{\pi}
\end{array}\right)\left(\begin{array}{l}
u_{t} \\
\pi_{t}
\end{array}\right)=\mathbf{N}\left(\begin{array}{c}
u_{t} \\
\pi_{t}
\end{array}\right) \\
x_{t}=F_{\pi} \pi_{t}+A F_{\pi} u_{t}
\end{array}\right. \\
\Leftrightarrow\left\{\begin{array}{c}
\left(\begin{array}{c}
u_{t+1} \\
x_{t+1}
\end{array}\right)=\mathbf{N}^{-1}(\mathbf{A}+\mathbf{B F}) \mathbf{N}\left(\begin{array}{c}
u_{t} \\
x_{t}
\end{array}\right)+\mathbf{N}^{-1}\left(\begin{array}{c}
\varepsilon_{t} \\
0
\end{array}\right) \\
\left(\begin{array}{c}
u_{t} \\
\pi_{t}
\end{array}\right)=\mathbf{N}^{-1}\left(\begin{array}{c}
u_{t} \\
x_{t}
\end{array}\right) \\
\pi_{t}=\frac{1}{F_{\pi}} x_{t}-A \pi_{t}
\end{array}\right.
\end{gathered}
$$

One has:

$$
\mathbf{N}^{-1}(\mathbf{A}+\mathbf{B F}) \mathbf{N}=\left(\begin{array}{cc}
\rho & 0 \\
(1-\lambda) F_{\pi} A \rho & \lambda
\end{array}\right)
$$

Which is Gali (2015) representation of the solution:

$$
x_{t}=\lambda x_{t-1}+(1-\lambda) F_{\pi} A \rho u_{t-1}=\lambda x_{t-1}+\varepsilon \frac{\lambda}{\beta \lambda \rho-1} \rho u_{t-1}
$$

\section{Proposition A4: Inequality demonstration.}

One has the following inequalities

$$
\text { If } \begin{aligned}
1-\frac{1}{2}(\beta+1+\kappa)+-\sqrt{\frac{1}{4} \beta^{2}\left(1+\frac{1}{\beta}+\frac{\kappa}{\beta}\right)^{2}-\beta} & >0 \Leftrightarrow \sqrt{\frac{1}{4}(1+\beta+\kappa)^{2}-\beta}>\frac{1}{2}(-1+\beta+\kappa) \\
\frac{1}{4}(1+\beta+\kappa)^{2}-\beta & >\frac{1}{4}(-1+\beta+\kappa)^{2} \\
(1+\beta+\kappa)^{2}-(-1+\beta+\kappa)^{2} & =4(\kappa+\beta)>4 \beta \\
\kappa+\beta & >\beta \text { which is true. }
\end{aligned}
$$


Figure 7: Impulse response function of inflation with $q=1,0.5,10^{-7}$ and $q=0$ top curve.
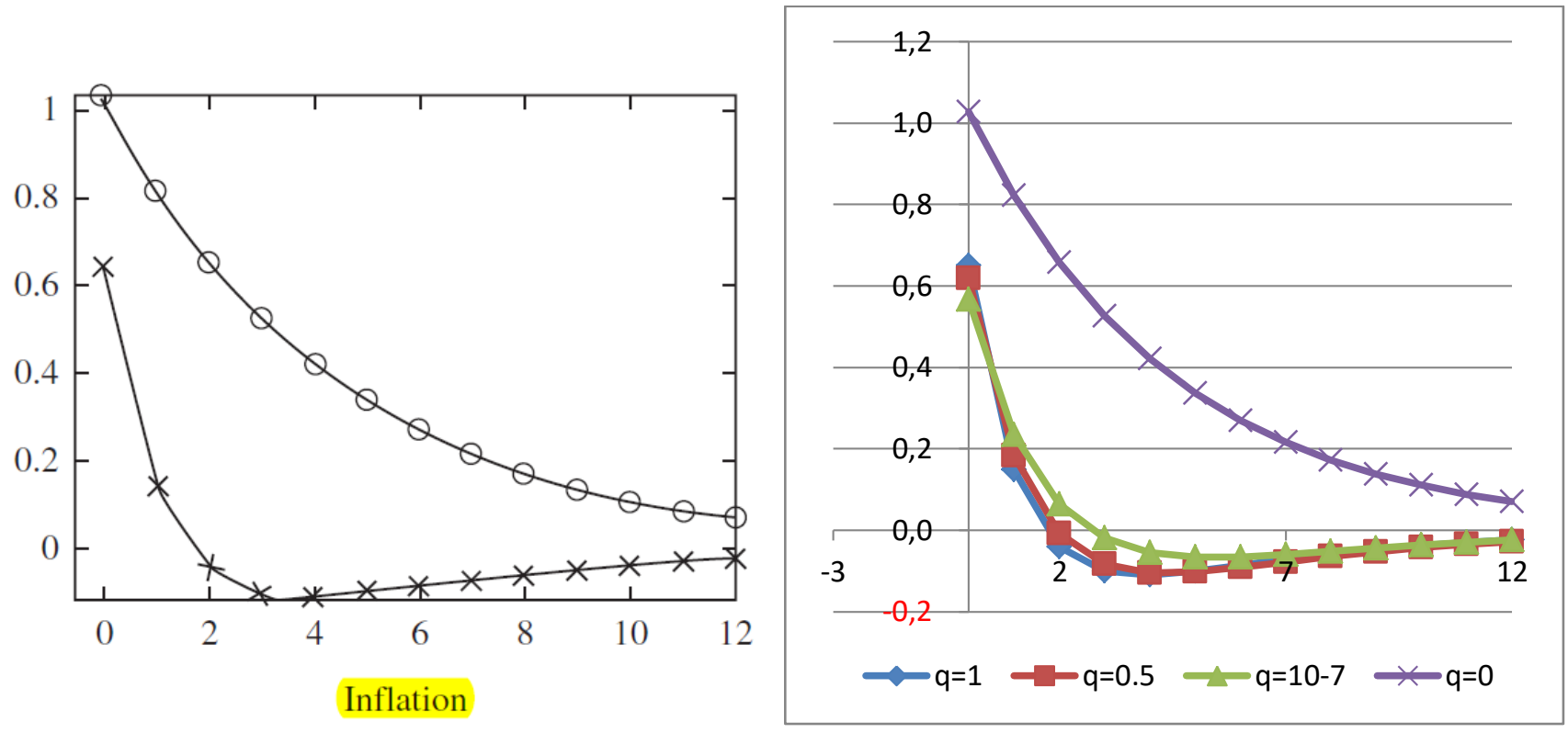

Figure 8 Impulse response of inflation: $q=10^{-7}$ and out of equilibrium path $+/-10 \%$ error.

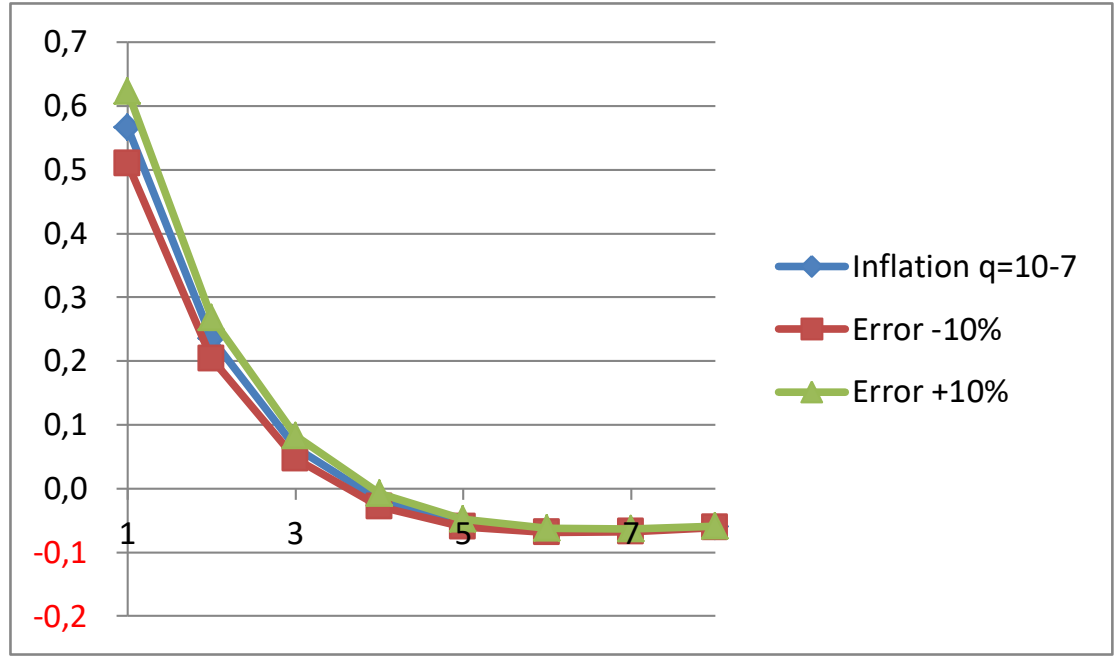

Figure 9. Impulse response of inflation: $q=0$ and out of equilibrium path $+/-10 \%$ error.

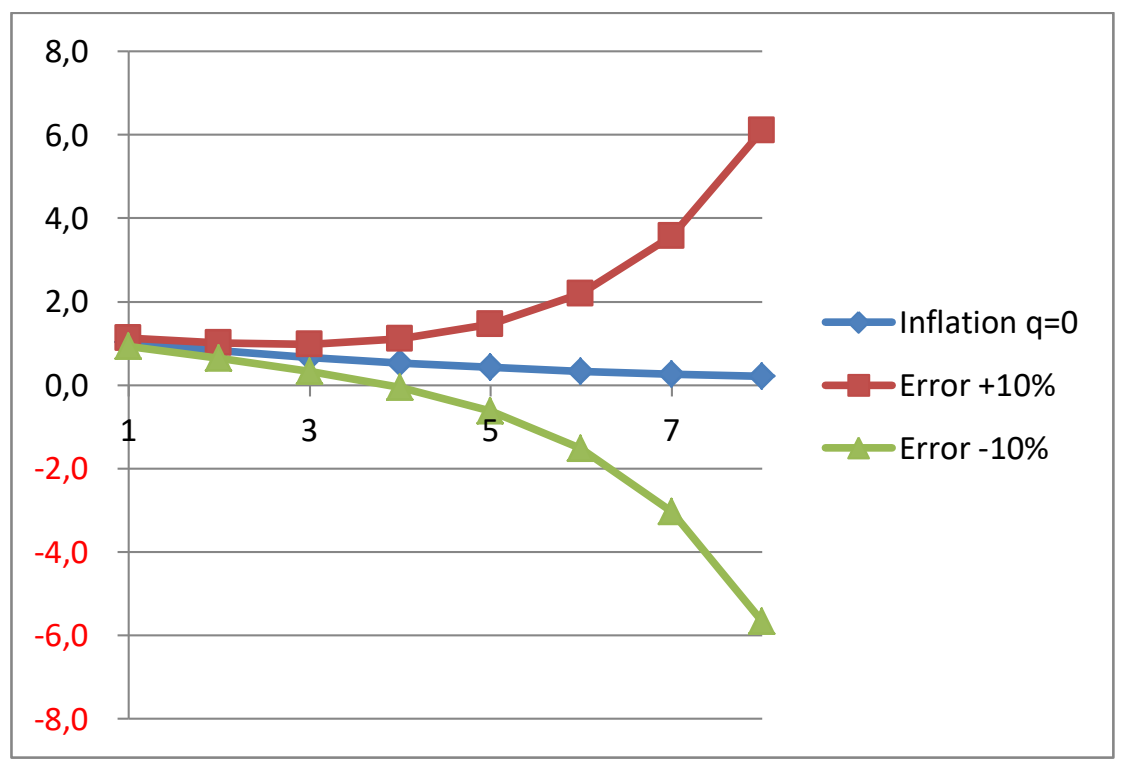

